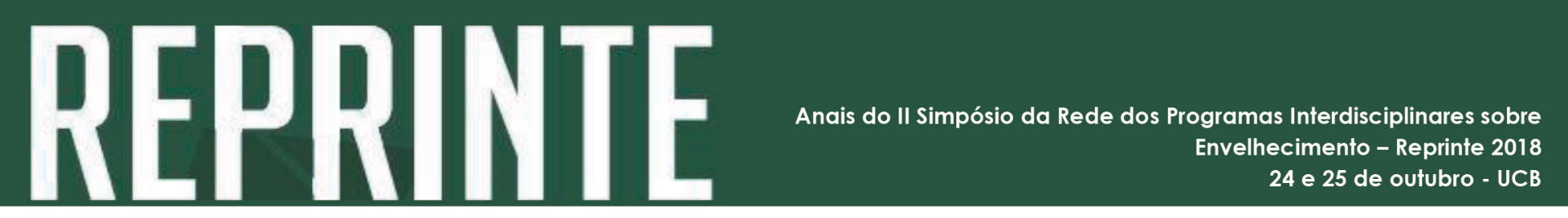

http://dx.doi.org/10.5335/rbceh.v16i1.9765

\title{
11) Crenças e atitudes sobre a velhice: infância, adolescência, avós e a intergeracionalidade
}

\author{
Jussara Soares Marques dos Anjos'; Lucy de Oliveira Gomes²; \\ Maria Liz Cunha de Oliveira²; Henrique Salmazo da Silva²
}

\section{Resumo}

A longevidade humana impõe novos desafios e oportunidades à configuração social e familiar da população brasileira. O objetivo deste estudo foi realizar uma revisão integrativa da literatura de artigos científicos de língua portuguesa sobre crenças e atitudes sobre a velhice em crianças e adolescentes no período de 2012 a 2017, com foco nas atividades intergeracionais e avosidade. Foram selecionados 14 artigos que atenderam aos critérios de inclusão e exclusão. Observou-se que as percepções e os significados de crianças e adolescentes sobre a velhice, em sua maioria foram positivas, associando-se a coabitação, conflitos intergeracionais, convivência com as pessoas mais velhas, e condições sóciodemográficas. As intervenções educacionais intergeracionais, oportunizam espaços de troca, reflexão e discussão de modo a minimizar os conflitos, estimular a convivência e desenvolver crenças e atitudes mais favoráveis sobre a velhice.

Palavras-chave: envelhecimento; atitude; avós; relações familiares, adolescente.

\section{Crenças e atitudes sobre a velhice}

As atitudes são descritas como opiniões de indivíduos e grupos em relação a objetos sociais, para as quais convergem sistemas afetivos e cognitivos. Têm um papel orientador, integrador e controlador no comportamento das pessoas, apresentando duas dimensões: a direção, manifestada em características opostas como positivo-negativo; e a intensidade, expressa em distintos graus (NERI, 1991).

No contexto da Gerontologia diversos estudos destacaram o papel das atitudes nas relações intergeracionais (PEREIRA et al, 2014; ARRAIS et al, 2012). Os achados sistematizados até o momento indicam que as atitudes e crenças são construídas em processos comuns de aprendizagem e são passíveis de mudanças, sendo a educação uma ferramenta central. Assim,

1 Mestranda no Programa de Pós-Graduação em Gerontologia na Universidade Católica de Brasília; Docente das Faculdades Integradas do Planalto Central - Curso de Enfermagem, Endereço para correspondência: Área Especial para Indústria Lote 2/3 - Setor Leste Gama, Brasília (DF) - CEP: 72445-020, Brasil. E-mail: jussaraanjos@gmail.com

2 Professores no Programa de Pós-Graduação em Gerontologia da Universidade Católica de Brasília. E-mails (na ordem em que aparecem): lucygomes2006@hotmail.com; lizcunhad@gmail.com; henriquesalmazo@gmail.com 
a Gerontologia Educacional tenta oportunizar espaços de troca e aprendizado entre as gerações para o exercício e para a formação de novos papéis sobre a velhice e o envelhecimento (FERRIGNO, 2010; TARALLO et al, 2016; ALVES; VIANNA, 2010; ZANON et al, 2011), com resultados promissores tanto para os idosos quanto para crianças e adolescentes.

Apesar dos estudos já documentados, existem variabilidades metodológicas e de interesses de pesquisa que precisam ser mais bem elucidadas para o delineamento de intervenções educacionais no Brasil. Nesse contexto, o objetivo deste estudo é realizar uma revisão integrativa da literatura, por meio da seleção e análise de artigos científicos que investigaram crenças e atitudes de crianças e adolescentes sobre a velhice entre o período de 2012 a 2017, com foco nas atividades intergeracionais e avosidade. As questões que nortearam a revisão foram: Qual o impacto das atividades intergeracionais sobre as crenças e atitudes sobre a velhice em crianças e adolescentes? O papel dos avós e as relações estabelecidas entre as gerações influenciam ou moderam as crenças e atitudes sobre a velhice em crianças e adolescentes? Para isso os estudos foram categorizados e realizou-se discussão dos temas e aspectos metodológicos empregados.

\section{Material e Métodos}

Trata-se de uma revisão integrativa da literatura a respeito das crenças e atitudes de crianças e adolescentes sobre a velhice. Para isso os estudos foram categorizados e discutidos com relação ao perfil da amostra, método, tipo de escala e avaliação utilizada, resultados principais e discussão; propiciando a construção dos percursos metodológicos, temas de interesse e a sistematização da literatura nacional (SOUZA et al, 2010; MENDES et al, 2008).

A busca dos artigos deu-se através dos descritores contidos no DeCS (Descritores em Ciência da Saúde), e inseridos por meio dos operadores booleanos na base de dados Bireme. Foram eles: Adolescente AND Envelhecimento, Atitude AND Criança AND Envelhecimento, Avós, Envelhecimento AND Criança, Relações familiares AND avós. Os artigos foram selecionados por esses descritores, e em seguida pelo título, resumo e por fim pela sua leitura integral.

Os critérios de inclusão foram: atender à combinação dos descritores; e ser artigo disponível na íntegra e gratuito, e publicado na língua portuguesa, no período de 2012-2017. Foram critérios de exclusão: artigos duplicados, artigos que não abordavam a temática da pesquisa e revisões sistemáticas da literatura.

\section{Resultados/Conclusões}

Dos 14 estudos analisados, quatro abordaram o impacto das atividades intergeracionais $(28,6 \%)$; três abordaram avosidade $(21,42 \%)$; dois abordaram avosidade e intergeracionalidade (14,3\%); dois analisaram relações entre crença, atitude, avosidade e intergeracionalidade (14,3\%); e os outros três abordaram, respectivamente: crença, atitude e avosidade $(7,1 \%)$; crença, atitude e intergeracionalidade $(7,1 \%)$; e crença e atitude $(7,1 \%)$.

Observou-se que as amostras investigadas foram heterogêneas, sendo compostas por crianças (de 3 a 10 anos) (42,8\%), adolescentes (21,1\%), adultos profissionais da educação, 
pessoas idosas e públicos mistos (50\%). Doze, dos 14 estudos, investigaram crianças e adolescentes, com faixas etárias específicas e empregaram metodologias voltadas à faixa etária investigada, adequando os materiais e instrumentos. Metade das pesquisas foram desenvolvidas no âmbito de contextos educacionais em escolas $(35,7 \%)$ e universidades $(14,3 \%)$, e o restante na comunidade e domicílio $(28,6 \%)$ ou em contextos mistos que envolviam comunidade, ambientes de educação e domicílio $(21,4 \%)$.

No que se refere à avaliação, foram aplicados instrumentos, entrevistas, rodas de conversa, cartas, imagens e desenhos. Observou-se que cinco dos 14 estudos mensuraram as crenças e atitudes em relação à velhice utilizando o Inventário de Crenças e Atitudes em Relação à Velhice (NERI, 1991; 1995). As demais avaliações utilizaram métodos qualitativos ou quantiqualitativos, com instrumentos elaborados pelos próprios pesquisadores.

Apesar da variabilidade dos instrumentos e das diferentes amostras e locais de avaliação disso, dez dos 14 estudos encontraram a prevalência de mais crenças e atitudes positivas em relação à velhice do que negativas; e as atitudes negativas, quando presentes, foram pontuais, ligadas à dependência, alterações cognitivas do idoso, baixa renda familiar, quantidade de pessoas no domicílio e tempo de convívio diário com idosos com alterações cognitivas (PEREIRA et al, 2014; GVOZD; DELLAROZA, 2012; OLIVEIRA et al, 2015; LUCHESI et al, 2012a; 2012b).

Observou-se que as crenças e atitudes de adolescentes e crianças de estudos publicados em língua portuguesa (Brasil e Portugal) foram influenciadas pelo papel dos avós no contexto familiar e pelas relações estabelecidas com os avós (PEREIRA et al, 2014; GVOZD; DELLAROZA, 2012; OLIVEIRA et al, 2015; LUCHESI et al, 2012a; 2012b) e pelos resultados de atividades intergeracionais (PIOVEZAN et al, 2015).

Apesar dos achados promissores, verifica-se que a inserção da Gerontologia Educacional ainda é um desafio na pesquisa e nos contextos de aprendizagem em nosso meio, especialmente devido à falta de condições estruturais e relativas à formação de recursos humanos (VIEIRA, 2012).

Além disso, conforme a variabilidade metodológica, é necessária padronização das avaliações, a fim de se verificar a eficácia dos programas de educação. Sugere-se a aplicação de instrumentos já validados e adaptados para a realidade brasileira (NERI, 1995; 1991; TODA$\mathrm{RO}, 2017)$.

A partir dos achados apresentados e de revisões bibliográficas (COELHO et al, 2016; COELHO et al, 2017; DEUS; DIAS, 2016; OLIVEIRA; PINHO, 2013; OLIVEIRA; KARNIKOWSKI, 2012), destaca-se a necessidade de investigar a avosidade e o impacto dos papéis dos avós.

Pode-se considerar, então, que a principal contribuição desta revisão foi apresentar e analisar os estudos de língua portuguesas mais recentes, publicados no período de 2012 a 2017, e que focalizaram estudos quantitativos e qualitativos a respeito das crenças e atitudes de crianças e adolescentes sobre a velhice. Em conjunto, esses dados podem contribuir para o delineamento de intervenções educacionais e paralelamente subsidiar outros estudos na área. 


\section{Beliefs and attitudes about old age: childhood, adolescence, grandparents and intergenerationality}

\section{Abstract}

Human longevity imposes new challenges and opportunities on the social and family configuration of the Brazilian population. The objective of this study was to carry out an integrative review of the literature of Portuguese - language scientific articles on beliefs and attitudes about old age in children and adolescents from 2012 to 2017, focusing on intergenerational activities and avoirability. We selected 14 articles that met the inclusion and exclusion criteria. It was observed that the perceptions and meanings of children and adolescents on old age were mostly positive, associating with cohabitation, intergenerational conflicts, coexistence with older people, and socio-demographic conditions. Intergenerational educational interventions provide opportunities for exchange, reflection and discussion in order to minimize conflicts, stimulate coexistence and develop more favorable beliefs and attitudes about old age.

Keywords: aging; attitude; grandparents; family relations, adolescent.

\section{Referências}

ALVES, Vicente Paulo; VIANNA, Lucy Gomes. Políticas públicas para a educação gerontológica na perspectiva da inserção social do idoso: desafios e possibilidades. Ensaio: avaliação e políticas públicas em educação, Rio de Janeiro, v. 18, n. 68, p. 489-510, jul./set., 2010. Disponível em: <http://www.redalyc.org/articulo.oa? id $=399537968005>$. Acesso em 01 ago., 2018.

ARRAIS, Alessandra da Rocha; BRASIL, Katia Cristina Tarouquella Rodrigues; CÁRDENAS, Carmen Jansen de; LARA, Luisa. O lugar dos avós na configuração familiar com netos adolescentes. Revista Kairós: Gerontologia, [S.l.], v. 15, n. 1, p. 159-176, mar., 2012. ISSN 2176-901X. Disponível em: <https://revistas.pucsp.br/index.php/ kairos/article/view/13111/9640>. Acesso em 20 jun., 2018.

COELHO, Maria Teresa Barros Falcão; DIAS, Cristina Maria de Souza Brito. Avós Guardiões: Uma Revisão Sistemática de Literatura do Período de 2004 a 2014. Psicologia: teoria e pesquisa. Brasília, v. 32, n. 4, p. 1-7, 2016. Disponível em: http://dx.doi.org/10.15900102.3772e324214. Acesso em 22 de jul., 2018.

COELHO, Sónia Margarida Santos; MENDES, Isabel Margarida Marques Monteiro Dias; RODRIGUES, Rogério Clemente. Grã-parentalidade: revisão integrativa da literatura. Revista Kairós: Gerontologia, [S.1.], v. 20, n. 1, p. 25-39, mar. 2017. Disponível em: <https://revistas.pucsp.br/index.php/kairos/article/view/2176-901X. 2017v20i1p25-39/22113>. doi:http://dx.doi.org/10.23925/2176-901X.2017v20i1p25-39. Acesso em 22 jul., 2018.

DEUS, Meiridiane Domingues de; DIAS, Ana Cristina Garcia. Avós cuidadores e suas funções: uma revisão integrativa da literatura. Pensando familias, v. 20, n. 2, p. 56-69, dez., 2016. Disponível em <http://pepsic.bvsalud.org/ scielo.php?script=sci_arttext\&pid=S1679-494X2016000200005\&lng=pt\&nrm=iso >. Acesso em 22 jul., 2018.

FERRIGNO, José Carlos. Coeducação entre gerações. 2 ed. Petrópolis, RJ: Vozes; São Paulo: SESC, 2010.233 p

GVOZD, Raquel; DELLAROZA, Mara Solange Gomes. Velhice e a relação com idosos: o olhar de adolescentes do ensino fundamental. Revista Brasileira de Geriatia e Gerontologia, Rio de Janeiro, v. 15, n. 2, p. 295-304, abr./jun., 2012. Disponível em: <http://www.redalyc.org/articulo.oa?id=403838796012>. Acesso em 20 jun., 2018.

LUCHESI, Bruna Moretti; DUPAS, Giselle; PAVARINI, Sofia Cristina Lost. Avaliação da atitude de crianças que convivem com idosos em relação à velhice. Revista Gaúcha de Enfermagem, v. 33, n. 4, p. 33-40, 2012a. Disponível em <http://www.scielo.br/scielo.php?script=sci_arttext\&pid=S1983-14472012000400004\&lng $=$ pt\&nrm =iso $>$. doi: http://dx.doi.org/10.1590/S1983-14472012000400004. Acesso em 20 jun., 2018. 
LUCHESI, Bruna Moretti; PAVARINI, Sofia Cristina Lost; VIANA, Aline Silveira. Alterações cognitivas de idosos no contexto domiciliar e atitudes de crianças em relação à velhice. Revista da Escola de Enfermagem da USP, v. 46, n. 2, p. 335-341, 2012b. Disponível em <http://www.scielo.br/scielo.php?script=sci_arttext\&pi$\mathrm{d}=\mathrm{S} 0080-62342012000200010 \& \mathrm{lng}=$ pt\&nrm =iso $>$. doi: http://dx.doi.org/10.1590/S0080-62342012000200010. Acesso em 20 jun., 2018.

MENDES, Karina Dal Sasso; SILVEIRA, Renata Cristina de Campos Pereira; GALVÃO, Cristina Maria. Revisão integrativa: método de pesquisa para a incorporação de evidências na saúde e na enfermagem. Texto \& Contexto-Enfermagem, v. 17, n. 4, p. 758-764, 2008. Disponível em: http://producao.usp.br/handle/BDPI/3509. Acesso em 01 ago., 2018.

NERI, Anita Liberalesso. Atitudes e crenças em relação à velhice. O que pensa o pessoal do SENAC - São Paulo. Relatório técnico. São Paulo: Senac, 1995.

NERI, Anita Liberalesso. Envelhecer num país de jovens. Significados de velho e velhice segundo brasileiros não idosos. Campinas: Unicamp, 1991. 155p

OLIVEIRA, Alessandra Ribeiro Ventura; KARNIKOWSKI, Margot Gomes de Oliveira. Apoio financeiro oferecido por avós a netos adolescentes. Revista Kairós: Gerontologia, [S.l.], v. 15, n. 1, p. 145-158, mar. 2012. Disponível em: $<$ https://revistas.pucsp.br/index.php/kairos/article/view/13113/9641>. Acesso em 22 jul., 2018.

OLIVEIRA, Alessandra Ribeiro Ventura; PINHO, Diana Lúcia Moura. Relações entre avós e seus netos adolescentes: uma revisão integrativa. Revista Brasileira de Geriatria e Gerontologia. Rio de Janeiro, v. 16, n. 3, p. 633-642, jul./set., 2013. Disponível em: <http://www.redalyc.org/articulo.oa?id=403838813019>. Acesso em 22 jul., 2018.

OLIVEIRA, Nathalia Alves de; LUCHESI, Bruna Moretti; INOUYE, Keika; BARHAM, Elizabeth Joan; PAVARINI, Sofia Cristina Lost. Avaliação da atitude das crianças que residem com idosos em relação à velhice. Acta Paulista de Enfermagem, v. 28, n. 1, 2015. Disponível em: http://wwrw.redalyc.org/articulo.oa?id=307035336015>. Acesso em 20 jun., 2018.

PEREIRA, Rafaelly Fernandes;DEFREITAS, Maria Célia;FERREIRA, Márcia deAssunção. Velhice para osadolescentes: abordagem das representações sociais. Revista Brasileira de Enfermagem, v. 67, n. 4, p. 601-9, jul./ago., 2014. Disponível em <http://www.scielo.br/scielo.php?script=sci_arttext\&pid=S0034=71672014000400601-\&lng=pt\&nrmiso >. Acesso em 20 jun., 2018. http://dx.doi.org/10.1590/0034-7167.2014670416.

PIOVEZAN, Marcelo; BESSA, Thaissa Araújo; BORGES, Felipe Souza Peito Silva; PRESTES, Stéfani Martins; CHUBACI, Rosa Yuka Sato. "Troca de cartas entre gerações": Projeto gerontológico intergeracional realizado em uma ILPI de São Paulo. Revista Kairós: Gerontologia, [S.l.], v. 18, n. 3, p. 137-153, set. 2015. Disponível em: <https://revistas.pucsp.br/index.php/kairos/article/view/26620/19042>. Acesso em: 20 jul., 2018.

SOUZA, Marcela Tavares; SILVA, Michelly Dias; CARVALHO, Rachel. Revisão integrativa: o que é e como fazer. Einstein, v. 8, n. 1 Pt 1, p. 102-6, 2010.

TARALLO. Roberta dos Santos; NERI, Anita Liberalesso; CACHIONI, Meire. Equivalência semântica e cultural da Intergenerational Exchanges Attitude Scale (IEAS). Revista Brasileira de Geriatria e Gerontologia, Rio de Janeiro, v. 19, n. 3, p. 453-463, jun., 2016. Disponível em: < http://www.scielo.br/scielo.php?script=sci_arttext\&pid=S1809-98232016000300453\&lng=en\&nrm=iso > . Acesso em 01 ago., 2018. http://dx.doi.org/10.1590/180998232016019.150002 .

TODARO, Monica de Ávila. Construção da Escala Todaro: atitudes de crianças em relação a idosos. Horizontes, v. 35, n. 1, p. 141-150, jan./abr., 2017. Disponível em: <https://revistahorizontes.usf.edu.br/horizontes/article/ view/313>. doi:https://doi.org/10.24933/horizontes.v35i1.313. Acesso em 22 jul., 2018.

VIEIRA, Sacha Lima. Relações intergeracionais: as barreiras da institucionalização. Revista Kairós: Gerontologia, [S.l.], v. 15, p. 119-133, fev. 2012. ISSN 2176-901X. Disponível em: <https://revistas.pucsp.br/index.php/kairos/ article/view/12782/9271>. Acesso em: 20 jul., 2018.

ZANON, Carla Bianca Ferreira Moncaio; ALVES, Vicente Paulo; CARDENAS, Carmen Jansen. Como vai a Educação Gerontológica nas Escolas Públicas do Distrito Federal? Um estudo com idosos e jovens. Revista Brasileira de Geriatria e Gerontologia, Rio de Janeiro, v. 14, n. 3, p. 555-566, 2011. Disponível em: http://www.redalyc.org/ articulo.oa?id=403834043014. Acesso em 01 ago., 2018. 